\section{Qualidade de vida e AIDS sob a ótica de pessoas vivendo com o agravo: contribuição preliminar da abordagem estrutural das representações sociais}

\author{
Quality of life and AIDS from the perspective \\ of persons living with HIV: a preliminary \\ contribution by the structural approach to social \\ representations
}

\author{
Calidad de vida y SIDA en personas infectadas: \\ contribución preliminar del enfoque estructural de \\ las representaciones sociales
}

\begin{abstract}
This descriptive qualitative study had the following objectives: identify the content and structure of social representations of quality of life and AIDS for persons living with the disease and analyze the structural relations between such representations. The sample included 103 persons with HIV in a municipality (county) in northern Rio de Janeiro State, Brazil. The methodology used free and hierarchical recall of words for the inductive terms "AIDS" and "quality of life for persons with AIDS", with analysis by the EVOC software. The probable core representation of AIDS was identified as: prejudice, treatment, family, and medications, with the same components identified for quality of life, plus healthy diet and work. We thus elaborated the hypothesis of joint, coordinated representational interaction, fitting the representations together, with implications for the symbolic grasp and quality of life for persons living with HIV. The findings provide backing for collective and individual health approaches to improve quality of life in this group.
\end{abstract}

Quality of Life; Acquired Immunodeficiency Syndrome; Social Psychology
Tadeu Lessa da Costa 1

Denize Cristina de Oliveira 2 Gláucia Alexandre Formozo 1

\section{Resumo}

Trata-se de estudo descritivo, qualitativo, tendo por objetivos: identificar o conteúdo e a estrutura das representações sociais da qualidade de vida e da AIDS entre pessoas com a doença e analisar as relações estruturais entre tais representações. Os sujeitos foram 103 pessoas soropositivas de município norte fluminense, Brasil. Empregou-se técnica de evocação livre e hierarquizada de palavras aos termos indutores "AIDS" e "qualidade de vida da pessoa que tem AIDS", com análise pelo software EVOC. Foram identificados, como provável núcleo central da representação da AIDS, preconceito, tratamento, família e medicações e, para a qualidade de vida, os mesmos componentes, acrescidos de boa alimentação e trabalho. Assim, elaborou-se hipótese de interação representacional do tipo coordenada, em conjunção, com encaixamento entre as duas representações, com implicações para a apropriação simbólica e avaliação da qualidade de vida pelas pessoas vivendo com a doença. Esses achados consistem em subsídios para abordagens coletivas e individuais em saúde para melhoria da qualidade de vida desse grupo.

Qualidade de Vida; Síndrome de Imunodeficiência Adquirida; Psicologia Social 


\section{Introdução}

A infecção pelo vírus da imunodeficiência humana (HIV) apresenta amplo espectro de apresentações clínicas, desde a fase aguda até seu estágio mais avançado, qual seja, de síndrome da imunodeficiência adquirida (AIDS), frequentemente, com contagem de células TCD4+ abaixo de $200 / \mathrm{mm}^{3}$ de sangue. Estima-se em torno de dez anos o tempo entre o contato infectante e o desenvolvimento da imunodepressão em sujeitos não tratados 1 .

Atualmente, são aproximadamente $34 \mathrm{mi}$ lhões de pessoas vivendo com HIV/AIDS no mundo, sendo considerado significativo o impacto da epidemia. Não obstante, observam-se avanços com a redução de sua incidência em termos globais, com 2,5 milhões de casos novos, em 2011, 20\% a menos que em 2001 2. No Brasil, entre 1980 e junho de 2012, foram identificados 656.701 casos de AIDS, seguindo o mesmo padrão de redução nas notificações 3 .

Vislumbram-se, também, mudanças no curso da infecção, com a transformação da AIDS de doença aguda e fatal em crônica 2 . Isso, sobretudo, pelo desenvolvimento da terapia antirretroviral altamente ativa (TARV) e sua ampla distribuição para pessoas vivendo com HIV/AIDS no país 4,5. Com isso, têm sido crescentes os estudos sobre a qualidade de vida desses sujeitos, pois o aumento na sobrevida não eliminou, mas adicionou novas facetas, como desafios psicossociais para uma vida melhor com o agravo 6 .

Nessa perspectiva, deve-se destacar que conhecer e compreender as condições psicossociais relacionadas às pessoas vivendo com HIV/ AIDS representa uma ferramenta importante para a abordagem delas no âmbito da atenção à saúde ${ }^{1}$. Isso porque diversos autores têm, de modo crescente, procurado abordar aspectos de natureza psicossocial, como informações, atitudes, sentimentos e crenças sobre a doença e o tratamento, autoestima e suporte social, ao estabelecerem a hipótese de seu papel, para além dos parâmetros biológicos clássicos simplesmente, na configuração da adesão terapêutica 7. A importância da consideração da possibilidade de influência dos referidos fatores é discutida, também, na constituição e percepção da qualidade de vida desse grupo 5,6,8. E, não obstante os debates e investimentos acadêmicos empreendidos no sentido de uma melhor análise desses aspectos, a necessidade da valorização deles, inclusive, já passou a figurar nas orientações das políticas públicas voltadas à assistência às pessoas vivendo com HIV/AIDS 1.

A abordagem da qualidade de vida, por sua vez, demanda, inicialmente, esforço conceitual, já que consiste em fenômeno complexo e multifacetado. Assim, a Organização Mundial da Saúde (OMS) 9 (p. 43), por meio de grupo de trabalho específico, definiu a qualidade de vida como: " $a$ percepção do indivíduo de sua posição na vida no contexto da cultura e sistema de valores nos quais ele vive e em relação aos seus objetivos, expectativas, padrões e preocupações. É um conceito de amplo espectro, que incorpora de modo complexo a saúde física, o estado psicológico, o nível de independência e as relações sociais das pessoas, bem como suas interfaces com importantes características de seu meio".

Desse modo, estudos têm apontado, como aspectos com repercussões para a qualidade de vida de pessoas vivendo com HIV/AIDS, o estigma 10; o acesso; a assistência pelos serviços de saúde e as relações com profissionais de saúde 11; trabalho e vínculos empregatícios 10; vivência da sexualidade 12; alterações corporais e percepção da autoimagem 13; e suporte social e relações familiares 14 .

Análises sobre a qualidade de vida das pessoas vivendo com HIV/AIDS têm sido desenvolvidas sobremaneira com abordagem quantitativa, com base em instrumentos padronizados e validados 4,9,10,12, mas se fazem presentes, também, iniciativas pautadas em referenciais teórico-metodológicos de natureza psicossocial. Na vertente psicossocial, encontram-se autores que desenvolveram pesquisas qualitativas em geral 15 e, também, trabalhos que empregaram a Teoria de Representações Sociais, delineando o modo pelo qual os sujeitos soropositivos se apropriam das informações sobre a doença e suas diversas dimensões no cotidiano 5 . Desse modo, os últimos trabalhos citados sinalizam que, nas representações sociais das pessoas vivendo com HIV/ AIDS, também ocorre a presença de elementos objetivos e subjetivos envolvidos no conceito da qualidade de vida 16 .

As relações sociais podem ser definidas como "uma forma de conhecimento socialmente elaborada e partilhada, com um objetivo prático, e que contribui para a construção de uma realidade comum a um conjunto social" 17 (p. 22). E, na perspectiva da abordagem estrutural da teoria de relações sociais, as representações seriam constituídas por dois sistemas complementares: um central, que conferiria seu significado, organização de seus conteúdos e maior estabilidade; e um periférico, o qual propiciaria integração do núcleo comum com as experiências de pessoas cotidianas e a proteção do sistema central, sendo, também, indicador de processos de mudança ${ }^{18}$.

Nesse ponto, julga-se necessário tecer algumas considerações acerca da possibilidade do 
estudo da qualidade de vida como objeto de relações sociais. Autores da abordagem estrutural das relações sociais referem que os objetos de representação têm, em comum, seu polimorfismo, pelo qual podem emergir em diferentes formas na sociedade 19. Destaca-se, ainda, uma saliência sociocognitiva em determinada cultura e tempo, bem como a existência de práticas, na população, focalizadas em direção a tais objetos sociais 20 .

Nesse sentido, a noção de qualidade de vida se faz notar no intercurso das comunicações e práticas sociais, sendo marcante sua característica polissêmica. Observa-se, também, que a abordagem desse objeto se faz presente tanto a partir do universo reificado quanto do consensual 21,22,23. Ocorre, portanto, o emprego do termo desde o senso comum, na linguagem cotidiana, da população em geral, jornalistas, políticos, por exemplo, até no campo da ciência, atravessando áreas como economia, sociologia, educação, medicina, enfermagem e psicologia 23.

Pode-se encontrar, na literatura, análise, inclusive, de registro noticioso em revista de circulação nacional direcionada ao público leigo com o tema qualidade de vida ${ }^{23}$. Salienta-se, então, que "a mídia exerce um importante papel na construção do senso comum, pois se encarrega de boa parte da exposição desse nas práxis humanas" 21 (p. 453). E, no caso das pessoas vivendo com HIV/AIDS, estudo anterior apontou para a relação direta entre as relações sociais da AIDS e da qualidade de vida, porém abordando-os como objetos sociais distintos 16 .

Ao considerar a qualidade de vida entre pessoas vivendo com HIV/AIDS sob o prisma das relações sociais, tendo, em vista, sua possível relação simbólica com as dimensões das próprias relações sociais da AIDS, torna-se interessante focalizar o desenvolvimento da abordagem estrutural das representações no campo da relação entre diferentes objetos e suas relações sociais 19,20. Trata-se de aspecto pouco explorado e com significativa relevância, pois não existe representação social isolada, estando toda representação relacionada com um conjunto de outras representações que constituem o ambiente histórico e social dos indivíduos 21.

Nessa perspectiva, apresentamos, como objeto para a presente pesquisa, as relações sociais da AIDS e da qualidade de vida com o agravo para as pessoas vivendo com HIV/AIDS. Constituem-se em objetivos: identificar o conteúdo e a estrutura das relações sociais da AIDS e da qualidade de vida entre pessoas vivendo com HIV/ AIDS e analisar as relações estruturais entre as relações sociais da AIDS e da qualidade de vida com a doença.
Somado aos aspectos que conferem relevância ao estudo, ressalta-se seu caráter original. Isso porque, em busca sistemática na Biblioteca Virtual de Saúde (BVS), incluindo diferentes bases, como LILACS e MEDLINE, aos termos "representações sociais" AND AIDS, foram identificadas 146 produções, porém não foram encontrados artigos acerca da estruturação interna das relações sociais da AIDS, bem como da qualidade de vida na perspectiva de pessoas vivendo com HIV/AIDS.

Assim, as publicações identificadas abordaram o conteúdo das referidas representações para esse grupo, restando lacuna quanto ao modo de organização interna dos componentes dessas relações sociais. E deve-se considerar que, de acordo com a abordagem estrutural das relações sociais, representações com conteúdos semelhantes podem ser diferentes se seus sistemas centrais assim o forem, dado que esses últimos determinam seu significado, organização e estabilidade 18 .

\section{Métodos}

Trata-se de estudo descritivo, com abordagem qualitativa, com referencial teórico da Teoria de Representações Sociais, especialmente a sua abordagem estrutural 18,19,20,24. Teve, como sujeitos, pessoas vivendo com HIV/AIDS, acompanhadas em Serviço de Atenção Especializada (SAE) em HIV/AIDS de município com médio porte da região norte do Estado do Rio de Janeiro, Brasil.

A amostragem dos sujeitos foi não probabilística, por conveniência, com os critérios de inclusão: ciência da soropositividade ao HIV; idade maior ou igual a 18 anos; estar no SAE no momento da coleta de dados; desejo de participar do estudo e estar em condições mentais que possibilitassem o fornecimento das informações necessárias. Assim, foram recrutados 103 sujeitos. Houve aprovação em Comitê de Ética em Pesquisa da Universidade do Estado do Rio de Janeiro, sob o registro 017.3.2011, respeitando-se o desejo das pessoas que vivem com HIV/AIDS quanto à participação no estudo, com disponibilização de Termo de Consentimento Livre e Esclarecido.

A coleta de dados ocorreu entre os meses de maio e outubro de 2011, abarcando todos os dias de atendimento na semana, de modo aleatório. Para tal, foram empregados um formulário com dados sociodemográficos e a técnica ou teste de evocações livres de palavras. O referido teste é importante e frequente ferramenta em pesquisas na abordagem estrutural das representações sociais, possuindo duas fases 18,19 . Na primeira, 
é demandado aos participantes que verbalizem palavras ou expressões que venham imediatamente à sua mente a partir de um termo indutor. O caráter espontâneo desse processo permite ter acesso rapidamente ao universo semântico do objeto estudado 18,19. Neste estudo, foram adotados dois termos indutores, que são: "AIDS" e "qualidade de vida da pessoa que tem AIDS", sendo orientada a produção de, no máximo, cinco itens para cada um desses termos.

Na segunda fase da técnica de evocações livres de palavras, é solicitado aos sujeitos que procedam à hierarquização das evocações livres realizadas em função da importância que é atribuída a elas para a definição de cada objeto social em questão 18,19,24. O registro das evocações foi efetivado pelo pesquisador em instrumento impresso específico.

O emprego da técnica de associação livre de palavras no campo das representações sociais é justificado, como em estudo de base de natureza semelhante, uma vez que essa técnica "permite ao mesmo tempo ter acesso ao conteúdo e sua hierarquização. Sua facilidade de utilização e de compreensão pelos sujeitos permite, igualmente, administrá-la a um grande número de participantes"19 (p. 249). Além disso, não obstante o histórico de emprego da referida técnica no campo de representações sociais, bem como em estudos, de um modo geral, na área da AIDS, pensase que possui potencial heurístico a tal propósito, sobretudo, se já ensejada a complexidade no bojo da análise das relações entre representações sociais, com apoio em aplicação de análise estrutural em investigação científica precedente 19 .

Nesse sentido, considera-se pertinente à técnica de coleta de dados adotada no presente trabalho o quantitativo de 103 participantes da pesquisa, tendo, em vista, o balizamento com estudos anteriores envolvendo pessoas vivendo com HIV/AIDS com abordagem processual das representações sociais 5,8 e, mesmo, com análise de evocações livres de palavras 16 . E, do ponto de vista operativo, destacam-se, também, dificuldades na coleta de dados junto a usuários no cotidiano dos serviços de saúde, como limite de espaço físico privativo, e adesão dos sujeitos à participação na pesquisa, seja pelos critérios de inclusão/exclusão ou tempo adicional necessário para a atividade.

A análise dos dados sociodemográficos ocorreu com auxílio do software SPSS, versão 17 (SPSS Inc., Chicago, Estados Unidos), e o escrutínio do produto da técnica de evocações livres de palavras pautou-se nos elementos teóricos da abordagem estrutural das relações sociais, com apoio nos cálculos pelo software Ensemble de Programmes Permettant l'Analyse des Evocations
(EVOC), versão 2005 (Laboratoire Méditerranéen de Sociologie/Centre National de la Recherche Scientifique/Université d'Aix-Marseille, Aix-enProvence, França). Desse modo, iniciou-se a análise das evocações livres com a padronização das palavras ou expressões evocadas pelos sujeitos para constituir-se um corpus. Isso se deu com a correção ortográfica, substituição das preposições e espaços por hífen, uniformização dos termos na forma plural ou singular e por gênero. Na sequência, buscou-se a homogeneização das evocações para redução da dispersão de seus conteúdos, classificando sob a mesma designação elementos com significação comum 7 .

Obteve-se, então, para cada termo indutor, um corpus de elementos e dois indicadores quantitativos para cada um destes últimos: frequência de evocação e a ordem média de importância (OMI) atribuída pelos sujeitos. O cruzamento entre frequência de evocação e a OMI de cada conteúdo representacional permite obter uma primeira identificação da organização da representação, considerando seus prováveis constituintes do sistema central e aqueles do sistema periférico. Para tanto, foram calculadas, também, a frequência média e a média das OMI (rang) para o conjunto das evocações em cada corpus 7,19 .

A referida análise das evocações possibilitou a construção de um quadro de quatro casas para cada termo indutor, englobando os conteúdos das representações sociais para os objetos sociais em questão, bem como a distribuição deles na estrutura representacional, segundo apontamentos da abordagem estrutural 18,19,20,21,22,23,24. Assim, o quadrante superior esquerdo, denominado zona do núcleo central, apresenta cognições mais frequentes e importantes. Esses itens podem ser centrais na representação, com estereótipos ou protótipos associados aos objetos em foco 18,24 .

No quadrante inferior esquerdo, chamado de zona de elementos de contraste, encontramse os termos enunciados por poucos sujeitos, mas avaliados como mais importantes para eles. Além disso, alguns subgrupos poderiam apresentar representações distintas do grupo como um todo, cujas cognições centrais estariam nessa área 18,24 .

No quadrante superior direito, zona da primeira periferia, são dispostos itens mais evocados, mas considerados menos importantes pelos participantes 18,19,20,21,22,23,24. São os componentes mais relevantes da periferia, podendo alguns de seus integrantes ser, eventualmente, centrais na representação ${ }^{25}$. No quadrante inferior direito, conhecido como zona da segunda periferia, estariam as cognições menos frequentes e 
menos importantes para a determinação do significado da representação, mas com maior relação com a inserção social e mais sensíveis às práticas imediatas dos sujeitos 18,19,20,21,22,23,24.

Finalmente, a análise da relação estrutural entre as representações sociais da AIDS e da qualidade de vida das pessoas vivendo com HIV/ AIDS se deu com base na apresentação visual do quadro de quatro casas para cada objeto social.

\section{Resultados}

O perfil sociodemográfico dos sujeitos revelou amostra com composição semelhante quanto ao sexo. A faixa etária predominante foi de 20 e 49 anos. A escolaridade mostrou composição majoritária de pessoas vivendo com HIV/AIDS com, ao menos, Ensino Fundamental completo, porém destacando-se quantitativo com segmento fundamental incompleto (Tabela 1).

A maioria dos sujeitos era casada, vivia com parceiro ou possuía união estável. A orientação sexual predominante declarada foi heterossexual. O tempo de diagnóstico de infecção pelo HIV mostrou maior quantitativo de pessoas vivendo com HIV/AIDS com 5 a 10 anos, seguido por $\geq$ 10,1 anos. Destacaram-se, de modo decrescente, os estágios 1 e 2 de evolução do agravo, segundo critérios do Centro de Controle e Prevenção de Doenças (Estados Unidos), com base no valor dos linfócitos TCD4+. Além disso, majoritariamente, os depoentes usavam a TARV (Tabela 1).

Em relação aos aspectos sociais e financeiros, houve distribuição semelhante entre pessoas vivendo com HIV/AIDS que trabalham e não trabalham. A renda individual predominante foi entre 1,1 e 3 salários mínimos, seguido por $\leq 1$ salário mínimo (Tabela 1).

No que diz respeito às representações sociais analisadas, houve a produção pelos sujeitos de 504 palavras ou expressões ao termo indutor AIDS, sendo 83 dessas diferentes. A frequência média foi calculada pelo software em 14,6, sendo aproximada para 15, e o rang foi de 2,97, ajustado para 3. Considerando os procedimentos e parâmetros enunciados, pôde-se elaborar, com auxílio do EVOC 2005, o quadro de quatro casas mostrado na Figura 1.

As cognições localizadas na zona do provável núcleo central das representações sociais da AIDS foram: preconceito; tratamento; família e medicações. Esses elementos, possivelmente centrais, parecem indicar ser a AIDS, para as pessoas vivendo com HIV/AIDS, um fenômeno que: gera preconceito, faz necessário o tratamento e as medicações, estando inserida a família nesse processo.
Tabela 1

Distribuição dos participantes da pesquisa segundo dados sociodemográficos e de saúde. Município do Norte do Estado do, Rio de Janeiro, Brasil, 2011.

\begin{tabular}{|c|c|c|}
\hline & f & $\%$ \\
\hline \multicolumn{3}{|l|}{ Sexo } \\
\hline Masculino & 55 & 53,4 \\
\hline Feminino & 48 & 46,6 \\
\hline \multicolumn{3}{|l|}{ Faixa etária (anos) } \\
\hline $20-29$ & 20 & 19,4 \\
\hline $30-39$ & 29 & 28,2 \\
\hline $40-49$ & 33 & 32,0 \\
\hline $50-59$ & 15 & 14,6 \\
\hline$\geq 60$ & 6 & 5,8 \\
\hline \multicolumn{3}{|l|}{ Escolaridade } \\
\hline Não estudou & 1 & 1,0 \\
\hline Fundamental incompleto & 34 & 33,0 \\
\hline Fundamental completo & 27 & 26,2 \\
\hline Médio completo & 31 & 30,1 \\
\hline Superior completo & 10 & 9,7 \\
\hline \multicolumn{3}{|l|}{ Estado marital } \\
\hline Solteiro/Sem parceiro fixo & 44 & 42,7 \\
\hline Casado/Vive com parceiro/União estável & 47 & 45,6 \\
\hline Parceiro fixo, mas não vive com ele & 12 & 11,7 \\
\hline \multicolumn{3}{|l|}{ Orientação sexual } \\
\hline Heterossexual & 79 & 76,7 \\
\hline Homossexual/Bissexual & 24 & 23,3 \\
\hline \multicolumn{3}{|l|}{ Tempo de diagnóstico (anos) } \\
\hline$\leq 1,9$ & 17 & 16,5 \\
\hline $2-5$ & 25 & 24,3 \\
\hline $5,1-10$ & 33 & 32,0 \\
\hline$\geq 10,1$ & 28 & 27,2 \\
\hline \multicolumn{3}{|l|}{ Uso de TARV } \\
\hline Sim & 84 & 81,6 \\
\hline Não & 19 & 18,4 \\
\hline \multicolumn{3}{|l|}{ Estágio do agravo (CD4/mm³) } \\
\hline Estágio 1 ( $\geq 500)$ & 43 & 41,7 \\
\hline Estágio 2 (499-200) & 39 & 37,9 \\
\hline Estágio $3(<200)$ & 8 & 7,8 \\
\hline Estágio 4 (valor desconhecido) & 13 & 12,6 \\
\hline \multicolumn{3}{|l|}{ Trabalha } \\
\hline Sim & 53 & 51,5 \\
\hline Não & 50 & 48,5 \\
\hline \multicolumn{3}{|l|}{ Renda individual (salários mínimos) } \\
\hline Sem renda & 17 & 16,5 \\
\hline$\leq 1$ & 30 & 29,1 \\
\hline $1,1-3$ & 36 & 35,0 \\
\hline $3,1-5$ & 10 & 9,7 \\
\hline$\geq 5$ & 10 & 9,7 \\
\hline Total & 103 & 100,0 \\
\hline
\end{tabular}

TARV: terapia antirretroviral. 
Figura 1

Quadro de quatro casas ao termo indutor "AIDS". Município do Norte do Estado do Rio de Janeiro, Brasil, 2011.

\begin{tabular}{|c|c|c|c|c|c|c|}
\hline OMI & $<3,0$ & & & $\geq 3,0$ & & \\
\hline $\begin{array}{l}\text { Frequência de } \\
\text { evocação média }\end{array}$ & Termo evocado & $\begin{array}{c}\text { Frequência de } \\
\text { evocação }\end{array}$ & OMI & Termo evocado & $\begin{array}{c}\text { Frequência de } \\
\text { evocação }\end{array}$ & OMI \\
\hline$\geq 15$ & $\begin{array}{c}\text { Preconceito } \\
\text { Tratamento } \\
\text { Família } \\
\text { Medicações }\end{array}$ & $\begin{array}{l}50 \\
22 \\
17 \\
17\end{array}$ & $\begin{array}{l}2,580 \\
2,136 \\
2,176 \\
2,706\end{array}$ & $\begin{array}{l}\text { Morte } \\
\text { Tristeza }\end{array}$ & $\begin{array}{l}22 \\
17\end{array}$ & $\begin{array}{l}3,864 \\
3,176\end{array}$ \\
\hline$<14$ & $\begin{array}{c}\text { Cura } \\
\text { Cuidados com a saúde } \\
\text { Força de vontade } \\
\text { Solidariedade } \\
\text { Adoecimento }\end{array}$ & $\begin{array}{c}14 \\
13 \\
12 \\
11 \\
8\end{array}$ & $\begin{array}{l}2,929 \\
2,538 \\
1,583 \\
2,182 \\
2,875\end{array}$ & $\begin{array}{l}\text { Sofrimento } \\
\text { Medo } \\
\text { Rejeição } \\
\text { Ruim } \\
\text { Sigilo } \\
\text { Depressão } \\
\text { Vergonha } \\
\text { Viver } \\
\text { Isolamento }\end{array}$ & $\begin{array}{l}14 \\
12 \\
11 \\
10 \\
10 \\
9 \\
9 \\
9 \\
8\end{array}$ & $\begin{array}{l}3,357 \\
3,500 \\
3,182 \\
3,400 \\
3,200 \\
3,333 \\
3,222 \\
3,000 \\
3,375\end{array}$ \\
\hline
\end{tabular}

OMI: ordem média de importância.

No que diz respeito aos elementos da zona de contraste, localizados no quadrante inferior direito, foram identificados os elementos: cura; cuidados com a saúde; força de vontade; solidariedade e adoecimento.

Na composição da primeira periferia, no quadrante superior direito das representações sociais da AIDS para as pessoas vivendo com HIV/ AIDS, foram evidenciadas as cognições morte e tristeza, as quais consistem em conteúdos negativos nesse contexto representacional. As suas frequências foram, respectivamente, 22 e 17, próximo ao registrado dos demais elementos do provável núcleo central, exceto o preconceito. $\mathrm{E}$ a OMI de tristeza teve valor próximo ao ponto de corte $(3,176)$.

Finalmente, no quadrante inferior direito, referente à segunda periferia, foram constatados os termos, segundo sua ordem de frequência absoluta: sofrimento; medo; rejeição; ruim; sigilo; depressão; vergonha; viver e isolamento.

Quanto às representações sociais da qualidade de vida para as pessoas vivendo com HIV/ AIDS, houve a produção, pelos sujeitos, de 505 palavras ou expressões ao termo indutor AIDS, sendo 83 dessas diferentes. A frequência média calculada pelo EVOC 2005 foi de 17,5, aproximada para 18 , e o rang foi de 2,97, revisto para 3 .
Com base nesses parâmetros, pôde-se construir, com auxílio do referido software, o quadro de quatro casas mostrado na Figura 2.

No caso do presente objeto representacional, pode-se dizer que as prováveis cognições integrantes do núcleo central da representação são, por ordem decrescente de frequência: boa alimentação; tratamento; família; trabalho e medicações. Isso devido a sua localização no quadrante superior esquerdo.

A relação entre esses termos sugere que a qualidade de vida da pessoa vivendo com HIV/ AIDS estaria relacionada: à necessidade de alimentação adequada; ao tratamento, em que estariam inseridas, com destaque, as medicações; havendo especial influência, em sua definição, do contexto familiar e do trabalho no processo. Além disso, é possível perceber, também, que os elementos provavelmente centrais tratamento família e medicações, exceto pelo preconceito, coincidem com aqueles que figuraram na zona do núcleo central nas representações sociais da AIDS para os sujeitos da pesquisa.

Quanto ao quadrante inferior esquerdo, correspondente à zona de contraste, compuseramno as cognições, segundo ordem decrescente de aparição: vida normal; cuidados com a saúde; solidariedade e atendimento no setor saúde. 
Quadro de quatro casas ao termo indutor "qualidade de vida da pessoa que tem AIDS". Município do Norte do Estado do Rio de Janeiro, Brasil, 2011.

\begin{tabular}{|c|c|c|c|c|c|c|}
\hline OMI & $<3,0$ & & & $\geq 3,0$ & & \\
\hline $\begin{array}{l}\text { Frequência de } \\
\text { evocação média }\end{array}$ & Termo evocado & $\begin{array}{c}\text { Frequência de } \\
\text { evocação }\end{array}$ & OMI & Termo evocado & $\begin{array}{c}\text { Frequência de } \\
\text { evocação }\end{array}$ & OMI \\
\hline$\geq 18$ & $\begin{array}{c}\text { Boa alimentação } \\
\text { Tratamento } \\
\text { Família } \\
\text { Trabalho } \\
\text { Medicações }\end{array}$ & $\begin{array}{l}37 \\
24 \\
21 \\
21 \\
20\end{array}$ & $\begin{array}{l}2,568 \\
2,583 \\
1,952 \\
2,810 \\
2,600\end{array}$ & Preconceito & 29 & 3,000 \\
\hline$<17$ & $\begin{array}{l}\text { Vida normal } \\
\text { Cuidados com a saúde } \\
\text { Solidariedade } \\
\text { Atendimento no setor } \\
\text { saúde }\end{array}$ & $\begin{array}{l}16 \\
14 \\
14 \\
10\end{array}$ & $\begin{array}{l}2,688 \\
2,786 \\
2,929 \\
2,700\end{array}$ & $\begin{array}{c}\text { Não beber } \\
\text { Ruim } \\
\text { Amizade } \\
\text { Descanso } \\
\text { Rejeição }\end{array}$ & $\begin{array}{l}12 \\
12 \\
11 \\
11 \\
10\end{array}$ & $\begin{array}{l}3,083 \\
4,000 \\
3,545 \\
3,818 \\
3,400\end{array}$ \\
\hline
\end{tabular}

OMI: ordem média de importância.

Em relação ao sistema periférico, observa-se, no quadrante superior direito, ou seja, na primeira periferia da representação, a presença da cognição preconceito, a qual se mostrou saliente $\mathrm{e}$ com OMI próximo ao valor de corte. Por sua vez, na segunda periferia, foram identificados: não beber; ruim; amizade; descanso e rejeição.

\section{Discussões}

Na perspectiva da abordagem estrutural, as cognições do sistema central possuem especial interesse para as representações sociais em tela, uma vez que determinam o seu próprio significado e a organização dos demais elementos representacionais. Tais componentes teriam caráter consensual, definindo a homogeneidade do grupo e, ao mesmo passo, estando ligados à memória coletiva e à história dele 19,20,24.

Com base nas classificações das dimensões do núcleo central 24, observa-se, nas representações sociais da AIDS para as pessoas vivendo com HIV/AIDS, a coexistência de cognições de ordem normativa e funcional, sendo o primeiro caso representado pelo preconceito, e o segundo, pelo tratamento, a família e as medicações. Por outro lado, o provável sistema central das representações sociais da qualidade de vida para as pessoas vivendo com HIV/AIDS apresenta predomínio de dimensão funcional, pois os componentes identificados no quadrante correspondente parecem estar voltados a processos mais operativos, e sugere efeito da proximidade com o objeto social investigado 24 , associado à configuração da própria vida com a soropositividade ao HIV.

Considerando o cotejamento entre resultados sobre as representações sociais da AIDS para pessoas vivendo com HIV/AIDS, o presente achado difere quanto ao possível núcleo central daquele apontado em estudo anterior, em que, com base em evocações livres de palavras e análise fatorial de correspondência, cogitou-se a centralidade dos elementos doença, médico e medicação ${ }^{16}$. Isso porque, no presente trabalho, fez-se marcante a dimensão do preconceito, o mais saliente, mas, também, da família e do tratamento, mais prontamente evocados.

O preconceito no campo do HIV/AIDS consiste, então, em aspecto fundamental para análise e abordagem psicossocial das pessoas vivendo com HIV/AIDS por seu impacto simbólico e material cotidiano 5,6,8,15. Do ponto de vista representacional, sua presença marcante pode ser compreendida a partir de sua inserção em conteúdos muito "arcaicos" do pensamento social, denominados de thematha. Assim, estaria ligado 
à ideia da dialética puro $x$ impuro, à marcação social ou ao estigma e às interdições sobre o contato 26. Tal constituição arcaica, no caso da AIDS, ocorreu a partir de sua gênese, com a ancoragem de suas representações na vertente biológica sobre aquelas da sífilis (vista como poluição) 17,27, do câncer (como microprocesso de invasão) 27 e das grandes e devastadoras epidemias, reativando categoriais simbólicas relativas ao contágio, que tende a interferir na aproximação física, apoiado nos modos de transmissão inicialmente divulgados, como secreções sexuais e sangue 17,27. Em sinergia a isso, na vertente moral, a ancoragem se deu na relação de oposição entre a liberdade do sexo dito seguro e as virtudes da tradição social, em que se encontrou um novo cavalo de batalha, sustentado pela autoridade religiosa 17 .

A identificação dos componentes tratamento e medicações como provavelmente centrais traz importantes implicações. Em um primeiro plano, enseja a possibilidade de engajamento no controle do agravo com a participação do setor de saúde, tanto em sua faceta institucional quanto nas práticas profissionais, o que contribui para a coexistência com a doença 5,6,7,8 .

Em outro plano, consistem em elementos correspondentes a uma atitude mais positiva diante da doença, que, entre seus momentos iniciais, esteve fortemente associada à sua característica aguda e à ideia de morte 6,7 . Reforça-se, com isso, a hipótese de uma mudança representacional acerca da AIDS 5,6, que, no caso do presente estudo, evidencia o fenômeno para pessoas vivendo com HIV/AIDS. Tal transição se daria, sobretudo, pelo afastamento da cognição morte dos elementos organizadores dessas representações sociais, permitindo a introdução de outros com conotação distinta, como medicações e tratamento, que passariam a substituir o desfecho fatal imediato simbólica e originalmente ligado à AIDS 7. Tal achado é também relevante pela apresentação de dados empíricos a respeito nas representações sociais das pessoas vivendo com HIV/AIDS, com base no aporte teórico-metodológico da abordagem estrutural, haja vista que uma mudança representacional efetiva ocorre quando se altera o seu sistema central 24 .

No que diz respeito à família, trata-se de elemento frequentemente apontado pelas pessoas vivendo com HIV/AIDS em estudos sobre os impactos psicossociais do agravo. Assim, é apontada como componente na estrutura de suporte social às pessoas vivendo com HIV/AIDS, trazendo as atitudes positivas ou negativas em seu seio, implicações para a avaliação da qualidade de vida com a doença 5,6,14. Pode ocorrer, em algumas situações, a preocupação das pesso- as vivendo com HIV/AIDS de que a família não saiba do diagnóstico, devido a receio de reações negativas 5,6. É interessante notar a presença desse elemento na presente pesquisa na zona do núcleo central das representações da AIDS, já que, em outra investigação em representações sociais, ele apenas emergiu nas representações sociais da qualidade de vida 16 .

Nessa perspectiva, tendo, em vista, a análise comparativa entre as zonas do núcleo central das representações sociais da AIDS e da qualidade de vida, observa-se a presença de conteúdos comuns. Isso, exceto pelo preconceito, exclusivo no primeiro caso, e pelas cognições boa alimentação e trabalho, apenas no segundo campo representacional. Entretanto, considerando a base de cruzamento estatístico ou prototípica da técnica de análise das evocações livres pelo quadro de quatro casas na identificação do sistema central 25, deve-se salientar que a cognição preconceito pode também ser central nas representações sociais da qualidade. Essa possibilidade se deve ao fato de que o referido componente emergiu, no caso das representações sociais da qualidade de vida, na primeira periferia, porém com segunda maior frequência nessa representação $(n=29)$ e OMI limítrofe $(3,000)$.

Em relação aos conteúdos boa alimentação e trabalho, pode-se considerar que, além de sua exclusividade nas representações sociais da qualidade de vida, possuem caráter funcional no sistema central dessas representações. O primeiro elemento citado, com maior saliência ( $\mathrm{n}=37$, $\mathrm{OMI}=2,568$ ) parece refletir a importância que os sujeitos do estudo atribuem ao processo alimentar na sua qualidade de vida, devendo ter padrão saudável 15 .

O destaque conferido à alimentação possivelmente ocorre devido às associações entre o processo de tentativa de naturalização da vida com HIV/AIDS, que se dá de modo conjunto ao tratamento medicamentoso, bem como pela adoção de práticas capazes de auxiliar na manutenção da saúde 5 . Consiste em atitude positiva diante da qualidade de vida com a doença e emerge como importante faceta coadjuvante ao tratamento medicamentoso diante da infecção pelo HIV 15. Assim, a importância com que tal componente simbólico emerge em alguns estudos qualitativos anteriores 5,6,7,8,9,10,11,12,13,14,15 tem provável relação com sua posição na zona do núcleo central na presente pesquisa. Porém, salienta-se que investigações prévias 8,16 sobre as representações sociais da AIDS e da qualidade de vida entre pessoas vivendo com HIV/AIDS não identificaram esse conteúdo no campo representacional dos sujeitos. 
Quanto ao componente trabalho, o conjunto das evocações abarca a sua concepção como trabalho em si, sendo atividade produtiva, bem como as facetas de empregabilidade e oportunidade no mercado como algumas de suas possíveis repercussões psicossociais. No caso das pessoas vivendo com HIV/AIDS, deve-se considerar que a atividade laboral permite, além do acesso às condições materiais de existência, o desvio do pensamento das questões, muitas vezes, negativas da doença, para a ação produtiva. E, com isso, há também a geração de sentimento de utilidade e produtividade 28 . Esse elemento emergiu em outros estudos 5,8,15,16 como conteúdo relativo à qualidade de vida para pessoas vivendo com HIV/AIDS, o que pode ocorrer por sua provável inserção no sistema central dessa representação, como ora apontam os presentes dados.

Desse modo, a configuração evidenciada nas zonas do núcleo central das duas representações em análise permite a elaboração preliminar da hipótese de que as representações sociais da qualidade de vida da pessoa vivendo com HIV/ AIDS possam manter uma relação de dependência com as representações sociais da AIDS. Aproxima-se do que se denomina, estruturalmente, de relação de encaixe entre representações sociais 19,20,24. De modo mais específico, pode-se considerar a possibilidade da ocorrência do estabelecimento de uma relação representacional do tipo coordenada, em conjunção 19,24, e envolvendo o encaixamento da representação da qualidade de vida da pessoa vivendo com HIV/AIDS na representação da AIDS para esse grupo.

Assim, as relações entre as representações sociais podem, inicialmente, ser consideradas em termos verticais, haja vista o papel desempenhado pelas ideologias em sua configuração. A isso se denominaria de efeitos de campo. Haveria, também, a possibilidade de ocorrência de outro modo de interface entre as representações, em um mesmo plano do pensamento social, em termos de coordenação. No contexto dessas, seriam incluídas as análises sobre as similaridades e divergências do núcleo central em uma ou mais representações, sendo referidas como em conjunção 20.

Nesse sentido, existem três tipos de relação de conjunção, classificados quanto à composição e à constituição dos sistemas centrais de uma representação: reciprocidade; antinomia e encaixe ${ }^{19}$. Nesse terceiro tipo, pertinente ao presente estudo, haveria uma hierarquia ou dependência de um objeto social em relação a outro. As representações envolvidas nessa situação remeteriam aos mesmos valores, com os julgamentos normativos dos objetos "inferiores" em dependência do objeto "superior", diferenciando-se em sua dimensão funcional 19. Essa composição funcional, por sua vez, poderia ser observada nos novos conteúdos representacionais, conferindo especificidade ao objeto encaixado 24 .

Destaca-se que, não obstante o autor da abordagem estrutural das RS ter apontado, em trabalhos anteriores 24 , a apresentação da relação de encaixamento como a presença do objeto "superior" no sistema central do objeto "inferior", tal definição foi posta de modo mais amplo, em estudo recente do mesmo pesquisador junto a outros pares, no qual explorou a relação entre as representações sociais da velocidade, da limitação de velocidade e de um sistema automotivo automático de controle de velocidade (LAVIA) 19. Assim, embora não houvesse a configuração da presença dos próprios objetos em seus respectivos núcleos centrais, esses últimos autores abordaram a relação de encaixe no contexto de uma rede de representações, definida "como um conjunto de representações sociais estruturadas por valores comuns, atravessadas por temas comuns e partilhadas por um grupo em um meio social dado" 19 (p. 266).

O foco analítico, portanto, toma, em perspectiva, também, a lógica de estruturação representacional e a transversalidade, em seu interior, de valores e temas comuns, socialmente elaborados e partilhados, de modo que "os julgamentos normativos dos objetos inferiores não dependa deles mesmos, mas dos objetos superiores" 19 (pp. 243-4). Além disso, nesse caso, objetos inferiores teriam cognições adicionais de natureza funcional como diferenciais em seu sistema central em relação àquele dos objetos superiores, conferindo-lhes a especificidade 19 . Pensa-se que esse padrão parece corresponder aos achados do presente estudo, pois as cognições provavelmente centrais nas representações sociais da AIDS, preconceito; tratamento; família e medicações, foram identificadas no possível núcleo central das representações sociais da qualidade de vida, acrescido de elementos considerados mais funcionais boa alimentação e trabalho.

É importante salientar que, de modo pertinente a este trabalho, as representações sociais preexistentes exercem papel de ancoragem para novas representações sociais 19 . Isso se daria para o caso de objetos sociais encaixados, como, no presente caso, tem-se a hipótese de ocorrer para a qualidade de vida, em que ora se aponta a possibilidade de existir uma relação representacional de dependência e não autonomia com as representações sociais da AIDS. Pode consistir em uma etapa transitória no contexto do processo de possível estruturação de uma nova representação 19.

Esse processo estaria em acordo com a função de saber e interpretação da realidade de uma 
representação social. De tal modo, as representações sociais ditas "superiores" passariam, então, a determinar o conteúdo e a estrutura de toda nova representação encaixada, tendo um papel importante na gênese representacional. E a identificação da rede de representações envolvidas nesse processo poderia permitir prever, de algum modo, a forma e a orientação da nova representação 19. Considera-se essa, então, uma formulação que poderia subsidiar a compreensão da transversalidade dos temas ora identificados na zona do núcleo central das representações sociais da AIDS e da qualidade de vida. Isso, pois sendo, possivelmente, o segundo objeto dependente em sua dimensão normativa e em seu significado do primeiro tender-se-ia a uma configuração semelhante no modo de construção e de partilhamento dos referidos objetos entre as pessoas que vivem com HIV/AIDS.

Além disso, haja vista o processo de ancoragem das representações sociais, observa-se que, ao objeto novo se apoiar em um esquema anterior, ocorre uma mais fácil incorporação cognitiva do primeiro, mas trazendo possíveis transformações para o segundo 29 . Dessa maneira, deve-se considerar a capacidade dessa relação entre as representações sociais da AIDS e da qualidade de vida das pessoas vivendo com HIV/ AIDS trazerem à baila mudanças, provavelmente mais positivas sobre os modos de representação, também, da referida doença para esses sujeitos com o decorrer do tempo.

Pensa-se serem, possivelmente, as alterações representacionais a partir de elementos representacionais de conotação positiva, tendo, em vista, o conjunto dos conteúdos identificados na zona do núcleo central das representações sociais da qualidade de vida, bem como em sua zona de contraste, que pode consistir no sistema central a um subgrupo das pessoas vivendo com HIV/AIDS participantes da pesquisa. Como consequência, tem-se a possibilidade de construção progressiva de plano simbólico, que favoreça as adaptações psíquicas e materiais necessárias para a convivência com a doença, pelas pessoas vivendo com HIV/AIDS.

\section{Conclusões}

Este trabalho teve por objetivos identificar o conteúdo e a estrutura das representações sociais da AIDS e da qualidade de vida entre pessoas vivendo com HIV/AIDS, haja vista o escrutínio das relações estruturais entre as referidas representações. Isso com a perspectiva das implicações relacionais representacionais aos dois objetos sociais em questão.
Caracterizou-se o provável sistema central para as representações sociais da AIDS composto pelos conteúdos preconceito, tratamento, família e medicações. Por sua vez, o núcleo central das representações sociais da qualidade de vida das pessoas vivendo com HIV/AIDS mostrou composição com os elementos boa alimentação, tratamento, família, trabalho, medicações e, possivelmente, também, preconceito. Pôde-se, dessa maneira, apresentar a hipótese de uma relação representacional do tipo coordenada, em conjunção, com encaixamento da representação da qualidade de vida da pessoa vivendo com HIV/ AIDS na representação da AIDS para esse grupo. Hipótese essa definida a partir de elementos teóricos do campo da abordagem estrutural das representações sociais ainda não empregados em artigos científicos da área para a investigação das representações da AIDS e da qualidade de vida para as pessoas vivendo com HIV/AIDS.

Assim, considera-se que a relação representacional em questão apresenta implicações para a concepção e a avaliação da qualidade de vida por parte das pessoas vivendo com HIV/AIDS, sobremaneira condicionada pelas próprias representações sociais da AIDS. Porém, adicionalmente, considera-se a possibilidade de influência mútua entre os dois objetos sociais, em que a representação da qualidade de vida com o agravo para esse grupo possui potencial de alterações positivas sobre a representação da própria AIDS, em sinergia com mudanças que já têm sido apontadas na literatura, bem como na presente pesquisa, em curso provável em relação à ideia de morte.

Haja vista a potencialidade do constructo de qualidade de vida e da compreensão de sua apropriação simbólica pelos grupos sociais, nesse caso, por pessoas vivendo com HIV/AIDS, pensa-se que o conjunto de achados do estudo consiste em subsídios para abordagens coletivas e individuais em saúde para maior bem-estar psicossocial a esses sujeitos. Além disso, aponta para aspectos a serem contemplados na elaboração e análise de políticas públicas relacionadas à linha de promoção de qualidade de vida entre pessoas vivendo com HIV/AIDS.

Finalmente, pensa-se que este estudo não esgota as possibilidades de explorações diversas diante da complexidade envolvendo a construção e o partilhamento das representações sociais e de sua relação com as representações da AIDS. Entretanto, pode-se dizer que contribui para a abordagem dessas facetas oriundas do advento da doença e da recente e cada vez mais crescente possibilidade de convivência com ela. Poderse-á, assim, subsidiar também outras pesquisas na área. 


\section{Resumen}

Se trata de un estudio descriptivo, cualitativo, con los objetivos de: identificar el contenido y la estructura de las representaciones sociales sobre calidad de vida y SIDA entre personas con la enfermedad y analizar las relaciones estructurales entre estas representaciones. Los sujetos fueron 103 entre personas que vivían con VIH en el Norte Fluminense, Brasil. Se empleó una técnica de evocación libre y ordenada de palabras a partir de "SIDA" y "calidad de vida de la persona que tiene SI$D A$ ", mediante un análisis de EVOC. El probable núcleo de la representación del SIDA fue prejuicio, tratamiento, medicamentos y familia. Para la calidad de vida, fueron los mismos componentes, además de buena comida y trabajo. Así, se ha planteado la hipótesis de interacción coordinada de representación, junto a las representaciones, con sus implicaciones en la apropiación simbólica y evaluación de la calidad de vida de personas que viven con SIDA. Estos hallazgos conforman una base de apoyo para los enfoques individuales $y$ colectivos en salud, con el fin de mejorar la calidad de vida de este colectivo.

Calidad de Vida; Síndrome de Inmunodeficiencia Adquirida; Psicología Social

\section{Colaboradores}

T. L. Costa e D. C. Oliveira participaram da concepção do projeto de pesquisa, coleta, análise de dados e redação do artigo. G. A. Formozo colaborou na revisão crítica e redação do artigo.

\section{Referências}

1. Departamento de DST, Aids e Hepatites Virais, Secretaria de Vigilância em Saúde, Ministério da Saúde. Protocolo clínico e diretrizes terapêuticas para adultos vivendo com HIV/AIDS. Brasília: Ministério da Saúde; 2013.

2. Joint United Nations Programme on HIV/AIDS. Global report: UNAIDS report on the global AIDS epidemic 2012. Geneva: Joint United Nations Programme on HIV/AIDS; 2012.

3. Departamento de DST, Aids e Hepatites Virais, Secretaria de Vigilância em Saúde, Ministério da Saúde. Boletim Epidemiológico AIDS - DST 2012; Ano IX, no 1.

4. Campos LN, César CC, Guimarães MDC. Quality of life among HIV-infected patients in Brazil after initiation of treatment. Clinics 2009; 64:867-75.

5. Gomes AMT, Silva EMP, Oliveira DC. Social representations of AIDS and their quotidian interfaces for people living with HIV. Rev Latinoam Enferm 2011; 19:485-92.
6. Souto BGA. O HIV, seu portador e o tratamento antirretroviral: implicações existenciais. São Carlos: EdUFSCar; 2008.

7. Costa TL, Oliveira DC, Formozo GA, Gomes AMT. Análise estrutural das representações sociais da terapia antirretroviral entre pessoas que vivem com HIV/AIDS: possibilidades de convivência, normatividade e ressignificação. Psicol Saber Soc 2013; 2:104-14.

8. Castanha AR, Coutinho MPL, Saldanha AAW, Ribeiro CG. Aspectos psicossociais da vivência da soropositividade ao HIV nos dias atuais. Psico (Porto Alegre) 2006; 37:47-56.

9. The WHOQOL Group. The development of the World Health Organization quality of life assessment instrument (The WHOQOL). In: Orley J, Kuyken W, editors. Quality of life assessment: international perspectives. Heigelberg: Springer Verlag; 1994. p. 41-60. 
10. Costa TL, Oliveira DC. Qualidade de vida de pessoas com vírus da imunodeficiência humana e a interiorização: avaliação multidimensional. Rev Enferm UFPE on line 2013; 7:5866-75.

11. Formozo GA, Oliveira DC. Professional self-protection and nursing care for HIV patients: both party representation. Acta Paul Enferm 2009; 22:392-8.

12. Reis RK, Santos CB, Dantas RAS, Gir E. Qualidade de vida, aspectos sociodemográficos e de sexualidade de pessoas vivendo com HIV/AIDS. Texto Contexto Enferm 2011; 20:365-75.

13. Tsuda LC, Silva MM, Machado AA, Fernandes APM. Body changes: antiretroviral therapy and lipodystrophy syndrome in people living with HIV/ AIDS. Rev Latinoam Enferm 2012; 20:847-53.

14. Yadav S. Perceived social support, hope, and quality of life of persons living with HIV/AIDS: a case study from Nepal. Qual Life Res 2010; 19:157-66.

15. Meirelles BHS, Silva DMG, Vieira FMA, Souza SS, Coelho IZ, Batista R. Percepções da qualidade de vida de pessoas com HIV/AIDS. Rev RENE 2010; 11:68-76.

16. Castanha AR, Coutinho MPL, Saldanha AAW, Ribeiro CG. Avaliação da qualidade de vida em soropositivos para o HIV. Estud Psicol (Campinas) 2007; 24:23-31.

17. Jodelet D. Representações sociais: um domínio em expansão. In: Jodelet D, organizador. As representações sociais. Rio de Janeiro: EdUERJ; 2001. p. 17-44.

18. Abric J-C. La recherche du noyau central et la zone muette des représentations sociales. In: Abric J-C, editor. Méthodes d'études des représentations sociales. Ramonville Saint-Agne; 2003. p. 60-80.

19. Pianelli C, Abric J-C, Saad F. Rôle des representations sociales préexistantes dans les processus d'ancrage et structuration d'une nouvelle representation. CIPS 2010; 86:241-74.

20. Flament C, Rouquette M-L. Anatomie des idées ordinaires: comment étudier les représentations sociales. Paris: Armand Colin; 2003.
21. Coutinho MPL, Franken I. Qualidade de vida no serviço público de saúde: as representações sociais de profissionais da saúde. Psicol Ciênc Prof 2009; 29:448-61.

22. Almeida MAB, Gutierrez, GL, Marques R. Qualidade de vida: definições e conceitos. In: Almeida MAB, Gutierrez GL, Marques R, organizadores. Qualidade de vida: definição, conceitos e interfaces com outras áreas de pesquisa. São Paulo: Escola de Artes, Ciências e Humanidades, Universidade de São Paulo; 2012. p. 13-50.

23. Seidl EMF, Zannon CMLC. Qualidade de vida e saúde: aspectos conceituais e metodológicos. Cad Saúde Pública 2004; 20:580-8.

24. Abric J-C. A abordagem estrutural das representações sociais: desenvolvimentos recentes. In: Campos PHF, Loureiro MCS, organizadores. Representações sociais e práticas educativas. Goiânia: Editora UCG; 2003. p. 37-57.

25. Pecora AR, Sá CP. Memórias e representações sociais da cidade de Cuiabá, ao longo de três gerações. Psicol Reflex Crít 2008; 21:319-25.

26. Moscovici S. Preconceito e representações sociais. In: Almeida AMO, Jodelet D, organizadores. Interdisciplinaridade e diversidade de paradigmas em representações sociais. Brasília: Thesaurus; 2009. p. 17-34.

27. Sontag S. Doença como metáfora: AIDS e suas metáforas. São Paulo: Companhia das Letras; 2007.

28. Ferreira RCM, Figueiredo MAC, Souza LB. Trabalho, HIV/AIDS: enfrentamento e dificuldades relatadas por mulheres. Psicol Estud 2011; 16:259-67.

29. Vala J. Representações sociais e psicologia social do conhecimento do quotidiano. In: Vala J, Monteiro M, organizadores. Psicologia social. Lisboa: Fundação Calouste Gulbenkian; 2002. p. 457-502.

Recebido em 19/Out/2013

Versão final reapresentada em 19/Jun/2014

Aprovado em 29/Ago/2014 